\title{
The Potential and Limitations of
} Precision Oncology: Lessons Learned from Whole-Exome Sequencing in an Exceptional Response to Everolimus in Advanced Renal Cell Carcinoma

\author{
Amanda Pilling ${ }^{a} \quad$ Christopher Wee ${ }^{b} \quad$ Eliezer Bar-Meir ${ }^{b} \quad$ Gregory Dyson $^{c}$ \\ Ok Hwang $^{a}$ Nilesh Guptad Dhananjay Chitale ${ }^{d}$ Clara Hwang ${ }^{a}$ \\ aDepartment of Internal Medicine, Henry Ford Health System, Henry Ford Cancer Institute, \\ Detroit, MI, USA; ${ }^{b}$ Department of Medicine, Wayne State University School of Medicine, \\ Detroit, MI, USA; 'Department of Oncology, Population Statistics, Wayne State University \\ School of Medicine, Detroit, MI, USA; ${ }^{d}$ Department of Pathology, Henry Ford Health \\ System, Henry Ford Cancer Institute, Detroit, MI, USA
}

\section{Keywords}

Biomarker - Mammalian target of rapamycin inhibitor - Durable response

\begin{abstract}
Through elucidating the genetic mechanisms of drug sensitivity, precision medicine aims to improve patient selection and response to therapy. Exceptional responders are patients that exhibit exquisite and durable responses to targeted therapy, providing a rare opportunity to identify the molecular basis of drug sensitivity. We identified an exceptional responder to everolimus, an oral inhibitor of the mammalian target of rapamycin (mTOR) pathway, in a patient with advanced renal cell carcinoma. Through whole-exome sequencing on pretreatment and metastatic tumor DNA, we identified alterations in several mTOR pathway genes, with several mutations implicated in mTOR activation. Importantly, these alterations are currently not included in commercially available next-generation sequencing panels, suggesting that precision medicine is still limited in its ability to predict responses to mTOR-targeted therapies. Further research to discover and validate predictive biomarkers of response to everolimus and other targeted therapies is urgently needed. Given the rarity of patients with exceptional responses to targeted agents, cooperative efforts to understand the molecular basis for these phenotypes are essential.
\end{abstract}


Pilling et al.: Whole-Exome Sequencing from an Exceptional Response to Everolimus

\section{Introduction}

Advances in genomics and an expanded understanding of disease biology has transformed the therapeutic landscape of advanced renal cell carcinoma (RCC). Identification of mTOR signaling as a central element of RCC disease biology has led to the development and application of mTOR inhibitors for treatment of advanced disease [1-3]. Temsirolimus and everolimus, both analogs of rapamycin (rapalogs), allosterically inhibit mTOR complex 1 (mTORC1) and block mTOR activation. However, the clinical benefit of rapalogs is typically modest with reported progression-free survival $<6$ months. Despite limited benefit in unselected patients, there are multiple reports of outlier cases where patients demonstrate exceptional therapeutic response to these drugs.

Previous studies suggest that genomic alterations in mTOR pathway genes are potential determinants of mTOR inhibitor sensitivity in RCC [4-6]. Understanding the molecular basis of response is critical to establish predictive biomarkers for these therapies. In this study, we performed whole-exome sequencing (WES) of tumor DNA from a patient with advanced RCC who achieved durable disease control with everolimus.

\section{Case Report}

We identified a 60-year-old male patient with an exceptional response to everolimus. He presented initially with gross hematuria and flank pain. CT and MRI demonstrated a large 12-cm mass replacing the left kidney with invasion of the left psoas muscle. There was no evidence of metastatic disease. He underwent radical nephrectomy 4 weeks after presentation. On pathologic examination, he was confirmed to have RCC with clear cell and papillary features, Fuhrman grade 3, with gross involvement of the renal vein. Eight lymph nodes were examined with no evidence of nodal metastases. He was treated with adjuvant sunitinib on a clinical trial. The patient was unable to tolerate therapy, and sunitinib was discontinued after 1 month.

One year after nephrectomy, surveillance imaging demonstrated new pulmonary and hepatic lesions, as well as abnormal tissue in the left nephrectomy bed and left adrenal gland. Liver biopsy confirmed metastatic RCC. The patient was started on pazopanib as first-line therapy for metastatic disease. He had mild toxicity but did not require dose reduction or interruption. Ten weeks after starting pazopanib, a mixed response was seen on imaging. Although his pulmonary metastases were stable to slightly improved, his disease had clearly progressed in the liver (Fig. 1a, d). The patient was started on everolimus at this time.

Disease in the lung, liver, adrenal gland, and nephrectomy bed was significantly improved after 11 weeks of everolimus therapy. His lesions continued to regress at the 6-and 9-month time points and remained stable for 3 years (Fig. 1c-f). At that time, an MRI of the orbit was ordered to evaluate ptosis and proptosis of the right eye. A solitary site of metastatic progression was discovered in the right frontal sinus causing erosion of the calvarium with extension to the scalp, mass effect on the right frontal lobe, and extension to the right orbit and ethmoid sinus. The metastasis was surgically resected with pathologic examination confirming metastatic clear cell carcinoma. The resection was considered clinically complete, and no adjuvant radiation was recommended. Despite this recurrence, his other metastatic disease remained stable with only a sub-centimeter nodule in the lung and no disease detectable in the abdomen and pelvis. Given good tolerance and lack of progression elsewhere, everolimus therapy was continued.

Two years after resection, MRI was concerning for progression on the basis of several small areas of abnormal enhancement. He was treated with stereotactic radiosurgery to right nasal, right temporal, and right frontal dural lesions. There was no evidence of progression elsewhere, and he remained on everolimus. His disease remained stable for an additional 2 years. Imaging at

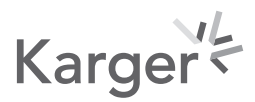



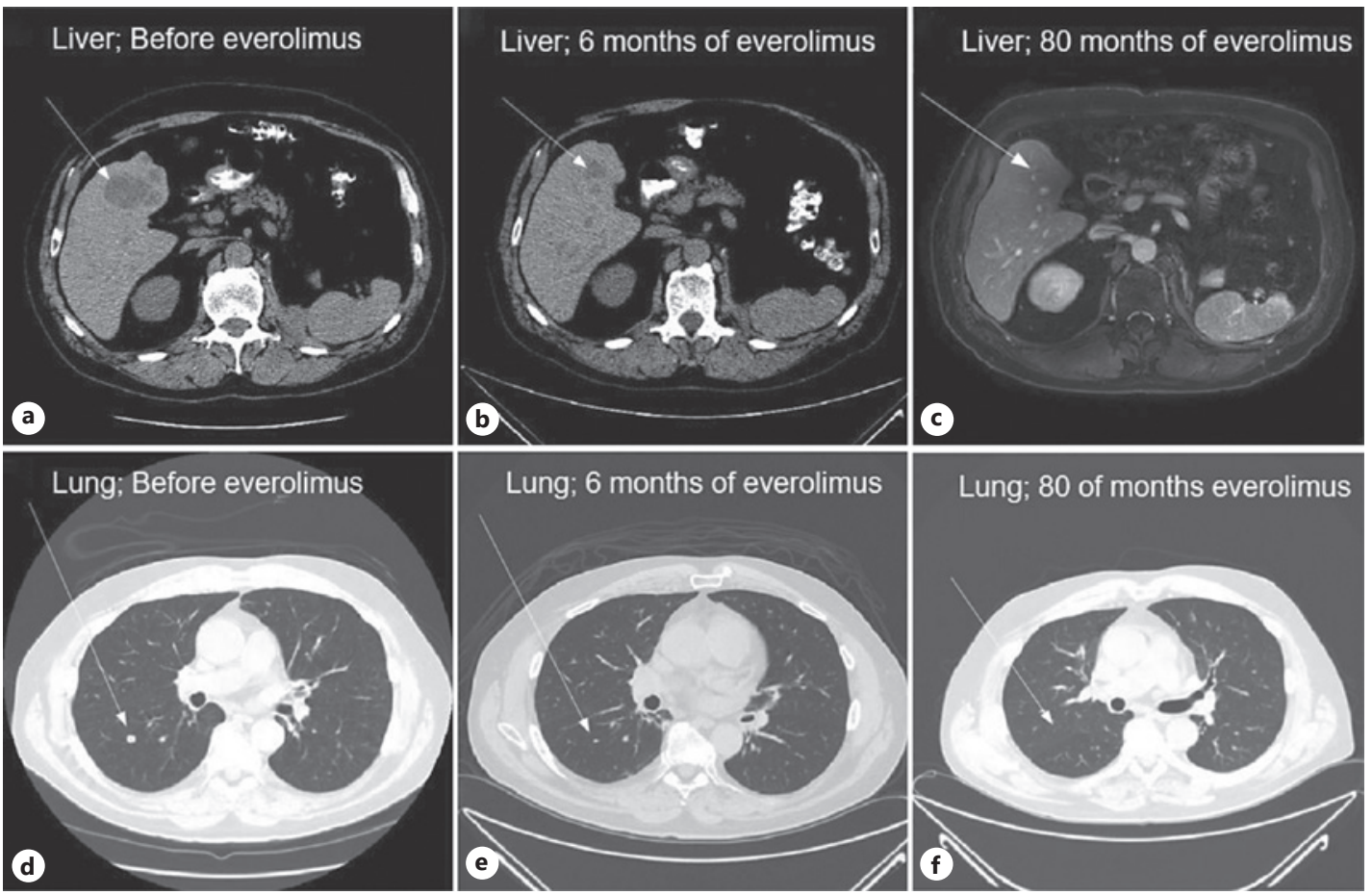

Fig. 1. Radiographic response to everolimus therapy. CT of the liver (a) and lung prior to starting everolimus (d); liver and lung after 6 months of everolimus (b, e); liver and lung after 80 months of everolimus (c, f). The metastatic lesions are indicated by white arrows.

that time demonstrated multiple lesions in the liver and spleen, as well as osseous metastatic disease. Everolimus therapy was discontinued, and the patient was started on nivolumab and denosumab. In total, the patient remained on everolimus for $>7$ years.

To identify potential molecular mechanisms for the extended benefit in response to everolimus, we performedWES on DNA from the primary (pretreatment) and metastatic tumor obtained at the time of surgical resection. The coding regions from approximately 25,000 genes were sequenced, with $90 \%$ of exons achieving $20 x$ coverage or greater. Analysis of mTOR pathway genes revealed variants in several genes associated with mTOR signaling (Table 1). The variants identified are present in the dbSNP (Single Nucleotide Polymorphism Database); however, only the PIK3C2G (P146L) mutation was found in the COSMIC (Catalogue of Somatic Mutations in Cancer) database. The alterations identified include 2 phosphoinositide 3-kinases (PI3Ks) that are involved in regulating Akt activation and missense mutations in PIK3R2 and PIK3C2G [7]. We also identified a missense mutation in diacylglycerol kinase zeta (DGKZ), encoding an enzyme that functions in mTORC1 activation [8]. In addition, we identified an alteration in protein kinase D (PRKD3) that activates Akt through diacylglycerol signaling [9]. A missense mutation was also detected in the insulin receptor (INSR) gene which encodes a receptor critical in activation of PI3K/Akt signaling and providing feedback regulation of mTORC1. Finally, we identified a missense mutation in eukaryotic translation initiation factor 3 (EIF3B) encoding an essential scaffolding component of the EIF3 complex activated by mTORC1 and functions in regulating protein translation [10].

To identify a potential genomic driver of disease recurrence in this patient, we compared alterations discovered in the primary tumor to those found in the metastatic lesion from the right frontal sinus obtained at the time of recurrence. Our analysis focused on alterations in mTOR pathway genes as a potential mechanism of everolimus resistance. Unique to the metastatic tumor are mutations in INSR, PIK3R2, and DGKZ genes, all of which are involved in either 
Table 1. Gene variants identified in WES analysis

\begin{tabular}{|c|c|c|c|c|c|}
\hline Gene & $\begin{array}{l}\text { Tumor } \\
\text { location }\end{array}$ & Mutation type & cDNA change & $\begin{array}{l}\text { Protein } \\
\text { change }\end{array}$ & Molecular function \\
\hline \multicolumn{6}{|c|}{ Genes in the mTOR pathway } \\
\hline EIF3B & $\mathrm{P}, \mathrm{M}$ & Missense & c. $190 \mathrm{~T}>\mathrm{C}$ & S64P & $\begin{array}{l}\text { Protein biosynthesis; activated by } \\
\text { mTORC1 to initiate translation }\end{array}$ \\
\hline PIK3C2G & $\mathrm{P}$ & Missense & c. $437 \mathrm{C}>\mathrm{T}$ & P146L & $\begin{array}{l}\text { PI3K catalytic subunit type II; promotes } \\
\text { prolonged activation of Akt2 }\end{array}$ \\
\hline PRKD3 & $\mathrm{P}$ & Missense & c. $1333 \mathrm{G}>\mathrm{T}$ & L445I & $\begin{array}{l}\text { Mediates DAG signaling; involved in Akt } \\
\text { activation and angiogenesis }\end{array}$ \\
\hline DGKZ & M & Missense & c. $29 \mathrm{C}>\mathrm{A}$ & A10D & $\begin{array}{l}\text { Transferase, lipid kinase; activates } \\
\text { mTORC1 through DAG metabolism }\end{array}$ \\
\hline INSR & M & Missense & c. $5 \mathrm{G}>\mathrm{C}$ & $\mathrm{A} 2 \mathrm{G}$ & $\begin{array}{l}\text { Metabolism; activates PI3K/Akt } \\
\text { signaling; negatively regulates mTORC1 }\end{array}$ \\
\hline PIK3R2 & M & Missense & c. $700 \mathrm{~A}>\mathrm{C}$ & S234R & $\begin{array}{l}\text { PI3K regulatory subunit ( } \mathrm{p} 85 \AA) \text {; } \\
\text { promotes constitutive activation of } \\
\text { PI3K/Akt }\end{array}$ \\
\hline \multicolumn{6}{|c|}{ Genes frequently altered in RCC } \\
\hline VHL & M & Missense & c. $266 \mathrm{~T}>\mathrm{A}$ & L89P & $\begin{array}{l}\text { Tumor suppressor; mutated form } \\
\text { induces stabilization of HIF-1a }\end{array}$ \\
\hline PBRM1 & M & Missense & c. $2626 \mathrm{G}>\mathrm{A}$ & R876C & $\begin{array}{l}\text { Likely tumor suppressor; subunit of } \\
\text { SWI/SNF chromatin remodeling } \\
\text { complex }\end{array}$ \\
\hline KDM5C & M & Splice site & $\mathrm{n} / \mathrm{a}$ & $\mathrm{n} / \mathrm{a}$ & $\begin{array}{l}\text { Histone demethylase; associated with } \\
\text { genomic rearrangement in tumors }\end{array}$ \\
\hline KDM3A & $\mathrm{P}, \mathrm{M}$ & Missense & c. $634 \mathrm{~A}>\mathrm{G}$ & $\mathrm{I} 212 \mathrm{~V}$ & $\begin{array}{l}\text { Histone demethylase; involved in } \\
\text { HIF-1a activation }\end{array}$ \\
\hline ARID4A & $\mathrm{P}$ & Missense & c. $2335 \mathrm{~A}>\mathrm{G}$ & T799A & $\begin{array}{l}\text { DNA binding protein; regulates several } \\
\text { transcription factors (i.e., RB, AR, E2F) }\end{array}$ \\
\hline
\end{tabular}

PI3K/Akt signaling or mTORC1 activation. Common between both the primary and metastatic tumor was the EIF3C missense mutation, suggesting a clonal mutation, occurring early in the evolution of the tumor.

In addition to mTOR pathway gene analysis, we also examined the sequencing data for genes commonly altered in RCC. In both the primary and metastatic tumors, we identified nonsynonymous alterations in $V H L, P B R M 1, K D M 3 A, K D M 5 C$, and ARID4A (Table 1). The alterations identified in $V H L$ and $P B R M 1$ have been previously characterized as inactivating, lossof-function mutations in RCC [11]. KDM3A, KDM5C, and ARID4A are involved in chromatin remodeling and are frequently altered in RCC [12].

\section{Discussion}

Emerging data on the role of mTOR signaling revealed that mTOR-activating genetic alterations occur frequently in cancer, suggesting that these tumors would be sensitive to MTOR inhibitors. Specifically, several studies have identified activating mutations in the MTOR gene, inactivating mutations in TSC1 or TSC2, and alterations in upstream effectors (i.e., PI3Kand PTEN), 
Table 2. mTOR pathway-associated genes included in analysis

\begin{tabular}{|c|c|c|c|c|c|}
\hline $\begin{array}{l}\text { PI3K/Akt } \\
\text { signaling } \\
\text { "upstream" }\end{array}$ & $\begin{array}{l}\text { PIK3CA, PIK3CB, } \\
\text { PIK3CD, PIK3CG, } \\
\text { PIK3C2, PIK3R1, } \\
\text { PIK3R2, AKT1/2/3, } \\
\text { PTEN, NF1, NF2 }\end{array}$ & $\begin{array}{l}\text { mTOR signaling } \\
\text { "upstream" }\end{array}$ & $\begin{array}{l}\text { MTOR, RPTOR, } \\
\text { RICTOR, } \text { LLST8, } \\
\text { FKBP12, DEPDC5, } \\
\text { RHEB, TSC1, TSC2 }\end{array}$ & $\begin{array}{l}\text { mTOR signaling } \\
\text { "downstream" }\end{array}$ & $\begin{array}{l}\text { RPS6K1, EIF3 } \\
\text { complex, EIF4 } \\
\text { complex, SREBP } \\
4 E B P, \text { HIF1, } \\
\text { AMPK, STK11 }\end{array}$ \\
\hline
\end{tabular}

all support high-level mTOR activation [4-6]. In RCC, alterations in MTOR, TSC1, or TSC2 have been reported in up to $10 \%$ of tumors, suggesting a potential link to mTOR inhibitor sensitivity. However, there is conflicting evidence in recent studies showing minimal association between mTOR pathway variants and response to mTOR inhibitor therapy [13,14]. Evaluating cases where patients demonstrate an extraordinary response to everolimus has the potential to identify the molecular mechanisms of sensitivity and advance the development of a predictive biomarker for advanced RCC.

In this case study, WES analysis was performed on tumor DNA from an RCC patient with extraordinary response to everolimus. This analysis included focused evaluation of mTOR pathway genes frequently altered in cancer and previously reported to be associated with response to mTOR inhibition (Table 2). The variants identified in our analysis have not been reported to be associated with sensitivity to MTOR inhibitors; however, several of them have been shown to activate oncogenic mTOR signaling. Specifically, PIK3R2 and PIK3C2G are frequently mutated in several malignancies where they have been shown to promote PTEN instability and constitutive PI3K/Akt activation $[15,16]$. We also identified mutations in INSR and $P R K D 3$, both regulating mTORC1 activity through PI3K/Akt signaling, and it is suggested that $P R K D 3$ genetic alterations can promote proliferation and survival signaling through prolonged Akt activation [17]. Finally, EIF3B expression has been linked to increased mTORC1 activation and was recently identified as a prognostic marker for adverse outcomes in ccRCC [10]. Importantly, the EIF3B variant was found in both the primary and metastatic tumors, suggesting a truncal alteration that occurred early in tumorigenesis.

Study of exceptional responders provides the opportunity to discover the molecular basis for sensitivity to anticancer therapies. Advances in DNA sequencing technology with ultrahigh-throughput next-generation sequencing (NGS) enables insight into the tumor-wide mutational landscape and has rapidly advanced the field of precision oncology. Employing the WES approach in super-responders facilitates the discovery of novel molecular mechanisms that can be used in developing a predictive marker for prospectively identifying patients who may similarly respond to the specific therapy. Commercially available NGS panels are often limited to known pathologic variants and those that predict response to targeted therapies. Importantly, most diagnostic and research NGS panels have a limited number of genes involved in mTOR signaling, therefore limiting applicability for discovery of a predictive marker for everolimus therapy. Through a WES approach, we identified several novel variants in mTOR pathway genes as potential molecular markers of everolimus sensitivity. Additional investigation is needed to characterize the functional significance of these alterations in everolimus sensitivity and apply these findings to future biomarker studies.

\section{Conclusion}

This case highlights the importance of molecular characterization in patients with exceptional response to targeted agents. WES has the capability to detect potential therapeutic targets not included in precision medicine panels that can be employed as a clinical tool in 
guiding therapy selection. The discovery of novel variants in a patient with an exceptional response to everolimus provides the evidence to warrant further investigation as potential predictive markers.

\section{Statement of Ethics}

Written informed consent was obtained from the patient for the publication of this case report and any accompanying images.

\section{Conflict of Interest Statement}

The authors declare no conflicts of interest.

\section{Funding Sources}

This work was supported by the Henry Ford Health System Physician Scientist grant (C.H.).

\section{Author Contributions}

C.W. and E.B.-M. compiled clinical data and imaging studies. C.W. and O.H. prepared study material for WES analysis. N.G. and D.C. performed pathological analysis and data analysis. D.C., G.D., A.P., and C.H. performed data analysis and interpretation. A.P. was involved in manuscript, table, and figure preparation. C.H. and G.D. were involved in conception and study design. All authors read and approved the final version of this manuscript.

\section{References}

1 Robb VA, Karbowniczek M, Klein-Szanto AJ, Henske EP. Activation of the mTOR signaling pathway in renal clear cell carcinoma. J Urol. 2007;177(1):346-52.

2 Hudes G, Carducci M, Tomczak P, Dutcher J, Figlin R, Kapoor A, et al. Temsirolimus, interferon alfa, or both for advanced renal-cell carcinoma. N Engl J Med. 2007;356(22):2271-81.

3 Motzer RJ, Escudier B, Oudard S, Hutson TE, Porta C, Bracarda S, et al. Efficacy of everolimus in advanced renal cell carcinoma: a double-blind, randomised, placebo-controlled phase III trial. Lancet. 2008;372(9637):449-56.

4 Iyer G, Hanrahan AJ, Milowsky MI, Al-Ahmadie H, Scott SN, Janakiraman M, et al. Genome sequencing identifies a basis for everolimus sensitivity. Science. 2012;338(6104):221.

5 Voss MH, Hakimi AA, Pham CG, Brannon AR, Chen YB, Cunha LF, et al. Tumor genetic analyses of patients with metastatic renal cell carcinoma and extended benefit from mTOR inhibitor therapy. Clin Cancer Res. 2014; 20(7):1955-64.

6 Kwiatkowski DJ, Choueiri TK, Fay AP, Rini BI, Thorner AR, de Velasco G, et al. Mutations in TSC1, TSC2, and MTOR are associated with response to rapalogs in patients with metastatic renal cell carcinoma. Clin Cancer Res. 2016;22(10):2445-52.

7 Vallejo-Diaz J, Chagoyen M, Olazabal-Moran M, Gonzalez-Garcia A, Carrera AC. The opposing roles of PIK3R1/ p85alpha and PIK3R2/p85beta in Cancer. Trends Cancer. 2019;5:233-44.

8 Torres-Ayuso P, Tello-Lafoz M, Mérida I, Ávila-Flores A. Diacylglycerol kinase- $\zeta$ regulates mTORC1 and lipogenic metabolism in cancer cells through SREBP-1. Oncogenesis. 2015;4:e164.

9 Roy A, Ye J, Deng F, Wang QJ. Protein kinase D signaling in cancer: a friend or foe? Biochim Biophys Acta Rev Cancer. 2017;1868(1):283-94.

10 Zang Y, Zhang X, Yan L, Gu G, Li D, Zhang Y, et al. Eukaryotic translation initiation factor 3b is both a promising prognostic biomarker and a potential therapeutic target for patients with clear cell renal cell carcinoma. J Cancer. 2017;8(15):3049-61.

\section{Karger'}


11 Sato Y, Yoshizato T, Shiraishi Y, Maekawa S, Okuno Y, Kamura T, et al. Integrated molecular analysis of clearcell renal cell carcinoma. Nat Genet. 2013;45(8):860-7.

12 Ricketts CJ, De Cubas AA, Fan H, Smith CC, Lang M, Reznik E, et al. The cancer genome atlas comprehensive molecular characterization of renal cell carcinoma. Cell Rep. 2018;23(1):313-e5.

13 Voss MH, Chen D, Reising A, Marker M, Shi J, Xu J, et al. PTEN expression, not mutation status in TSC1, TSC2, or mTOR, correlates with the outcome on everolimus in patients with renal cell carcinoma treated on the randomized RECORD-3 trial. Clin Cancer Res. 2019;25(2):506-14.

14 Nassar AH, Hamieh L, Gray KP, Thorner AR, Fay AP, Lasseter KD, et al. Mutations and response to rapalogs in patients with metastatic renal cell carcinoma. Mol Cancer Ther. 2020;19(2):690-6.

15 Cortes I, Sanchez-Ruiz J, Zuluaga S, Calvanese V, Marqués M, Hernández C, et al. p85beta phosphoinositide 3-kinase subunit regulates tumor progression. Proc Natl Acad Sci U S A. 2012;109:11318-23.

16 Margaria JP, Ratto E, Gozzelino L, Li H, Hirsch E. Class II PI3Ks at the intersection between signal transduction and membrane trafficking. Biomolecules. 2019;9(3):104.

17 Chen J, Deng F, Singh SV, Wang QJ. Protein kinase D3 (PKD3) contributes to prostate cancer cell growth and survival through a PKCepsilon/PKD3 pathway downstream of Akt and ERK 1/2. Cancer Res. 2008;68(10): 3844-53. 